\title{
Dynamic performance of existing double track railway bridges at resonance with the increase of the operational line speed
}

\author{
E.Moliner ${ }^{\mathrm{a}, *}$, M.D.Martínez-Rodrigo ${ }^{\mathrm{a}}$, P.Museros ${ }^{\mathrm{b}, \mathrm{c}}$ \\ ${ }^{a}$ Universitat Jaume I, Department of Mechanical Engineering and Construction, 12071 Castellón, Spain \\ ${ }^{b}$ Universitat Politècnica de València, Department of Continuum Mechanics and Structural Analysis, 46022 Valencia, Spain \\ ${ }^{c}$ Fundación Caminos de Hierro para la Investigación y la Ingeniería Ferroviaria, 28002 Madrid, Spain
}

\begin{abstract}
This article addresses the dynamic behaviour of double track simply supported bridges of short to medium span lengths (10 $\mathrm{m}<L<25 \mathrm{~m}$ ) belonging to conventional railway lines. These structures are susceptible to experience inadmissible levels of vertical vibrations when traversed by trains at high speeds, and in certain cases their dynamic performance may require to be re-evaluated in case of an increase of the traffic velocity above $200 \mathrm{~km} / \mathrm{h}$. In engineering consultancies, these structures have been traditionally analysed under the passage of trains at different speeds using planar models, neglecting the contribution of transverse vibration modes and also the flexibility of the elastomeric bearings. The study presented herein endeavours to evaluate the influence of these two aspects in the verification of the Serviceability Limit State of vertical accelerations, which is of great interest in order to guarantee a conservative prediction of the dynamic behaviour. In the present study, the dynamic response of representative slab and girder bridges has been evaluated using an orthotropic plate finite element model, leading to practical conclusions regarding the circumstances under which the above mentioned factors should be considered in order to adequately evaluate the transverse vibration levels of the deck.
\end{abstract} Keywords: railway bridges, elastomeric bearings, resonance, cancellation, vertical accelerations

\section{Introduction}

During the last decades the extensive construction of new high-speed lines in developed and developing countries as well as the increase of the operating train velocity in the existing ones (above $200 \mathrm{~km} / \mathrm{h}$ ) has risen the concern of scientists and engineers for the dynamic behaviour of railway bridges. The regular and repetitive nature of groups of train axle loads can induce resonance situations in these structures, a phenomenon that takes place when the time interval between the passage of repeated groups of loads is a multiple of one of the natural periods of the bridge. $\hat{\Psi}_{n}=1$

\footnotetext{
*Corresponding author. Tel.:+34964387468; fax:+34964728106

Email address: molinere@uji.es (E.Moliner)
}

Regarding vertical accelerations, short-to-medium span simply supported (S-S) bridges (span lengths ranging between 10 and $25 \mathrm{~m}$ ) are specially critical, and may experience considerably high amplifications of the acceleration levels due to resonance, entailing harmful consequences [1, 2]. Hence the Serviceability Limit State of vertical acceleration prescribed by Eurocode (EC) [3] $\left(3.5 \mathrm{~m} / \mathrm{s}^{2}\right.$ for ballasted tracks, to avoid ballast instability) is one of the most demanding requirements and becomes crucial for the design of railway bridges.

A number of conventional railway lines has been partially adapted for high-speed traffic. Some representative examples are the Madrid-Sevilla and Valencia-Barcelona railway lines in Spain, as well as the first European highspeed line, Paris-Lyon. When a line is upgraded and exist- 
ing bridges are unable to fulfil the Standards, the horizontal structures are sometimes replaced by new decks with higher transverse stiffness. Alternatively, the strenghtening procedure consists in a partial embedment of the abutmens, leading to a kind of portal frame or integral bridge [1]. On the other hand, a number of researchers have evaluated in the past years the possibility of applying passive control techniques [4, 5, 6, 6] that could avoid the deck replacement by increasing structural damping. These facts point out the importance of using accurate enough numerical models, able to realistically predict the vibration levels in the deck with reasonable computational costs. Superfluous refinements are to be avoided, since engineers will employ them as a tool for deciding (according to the standards) what is the most adequate retrofit solution from the economical and technical point of view in each particular case.

Traditionally, planar numerical S-S beam models are very common in literature (see [8, 9, 10]). These models appear to be valid for single track, non-skewed bridges, since the response of this type of structures at resonance is mainly governed by the first flexural mode [10]. Nevertheless the contribution of three-dimensional modes, such as the first torsion mode of the bridge, could be significant in double track decks, due to the eccentricity of the loaded track and the proximity between the first bending and the first torsion natural frequencies in short span bridges. The quick development of computational technologies and the versatility of numerical methods have promoted the use of three-dimensional models for investigation purposes in recent years (see [11, 12]), but their application for railway bridge dynamic analyses is still less frequent in engineering consultancies unless a singular structure is designed. An explanation to this tendency could be found in the regulations in force at each particular country. Eurocode 1 (EC1) [11], which will be adopted in most of the European countries in the near future, encourages the use of planar models when the frequency of the first torsion eigenform exceeds 1.2 times that of the first longitudinal bending mode (for non skewed beam or plate type decks on rigid supports).

Also EC establishes the minimum number of modes required for an accurate mode superposition analysis, which is a computationally efficient technique in structures with linear behaviour. In this regard EC recommends the consideration of the natural frequencies and the corresponding mode shapes up to the greater of (i) $30 \mathrm{~Hz}$, (ii) 1.5 times the frequency of the fundamental mode of vibration or (iii) the frequency of the third one. However, this limitation highlights a potential inconsistency: when the natural frequency of the first torsion mode is higher than 1.2 times the fundamental frequency but falls below this previous criteria, should or should not be taken into account. This matter has not been reported yet in the scientific literature and is one of the main issues analysed in the present study.

On the other hand, for practical purposes the vertical stiffness of laminated elastomeric bearings found in short simply supported bridges tends to be neglected by engineers for several reasons: in first place, these elements introduce spurious high-frequency oscillations in the predicted dynamic response, which can only be attenuated by including the track in the model or simulating its distributive effect at the entrance and exit of the loads using specific functions [6], thus further complicating the modelling task. Secondly, the elastomeric bearings are rather stiff, and are traditionally assumed rigid in the vertical direction for practical purposes. However, several studies show that their vertical stiffness may affect the dynamic response of the structure when subjected to railway traffic [13].

In the present contribution the authors have simulated the dynamic behaviour of S-S reinforced concrete slabs and pre-stressed concrete girder bridges belonging to conventional lines in which an increase of the maximum design velocity is envisaged. The numerical models adopted for 
this investigation follow purposely the main simplifications and tendencies adopted by engineers for practical applications (which are also in accordance with the European Standards) in a view to analyse their suitability. The selected case studies are intentionally restricted to double track bridges, since these are common structures in existing railway lines and may experience a significant contribution of the first torsion mode, with a natural frequency in the vicinity of the fundamental one. The results and conclusions presented herein provide an enhanced understanding of the importance of using three dimensional numerical models and of the effect of the elastic bearings for the assessment of the maximum acceleration levels in double track decks.

\section{Theoretical background}

The maximum dynamic response of a bridge under the circulation of trains is mainly conditioned by two of the classical phenomena related with the moving load problem: resonance and cancellation. For that reason, in a view to obtain a theoretical basis to enhance the understanding of the elastic supports effect on the dynamic response, these phenomena have been studied in a first approach by using the simplest beam model: a Bernoulli-Euler beam supported on vertical elastic supports (Fig. 1). The starting point of this investigation are the results published by the authors in [14], where the effect of the supports vertical stiffness on the frequency and amplitude of the resonant response of elastically supported (E-S) beams is analysed in detail.

The exact frequency equation and mode shapes of the E-S beam can be found in [15, 16], neglecting structural damping, shear deformation and rotary inertia effects. In this case the frequency equation is given by

$$
\begin{array}{r}
\left(\frac{\pi^{3}}{\kappa}\right)^{2}+\frac{\pi^{3}}{\kappa} \lambda^{3} \frac{\sinh (\lambda) \cos (\lambda)-\cosh (\lambda) \sin (\lambda)}{\sin (\lambda) \sinh (\lambda)} \\
+\lambda^{6} \frac{1-\cos (\lambda) \cosh (\lambda)}{2 \sin (\lambda) \sinh (\lambda)}=0
\end{array}
$$

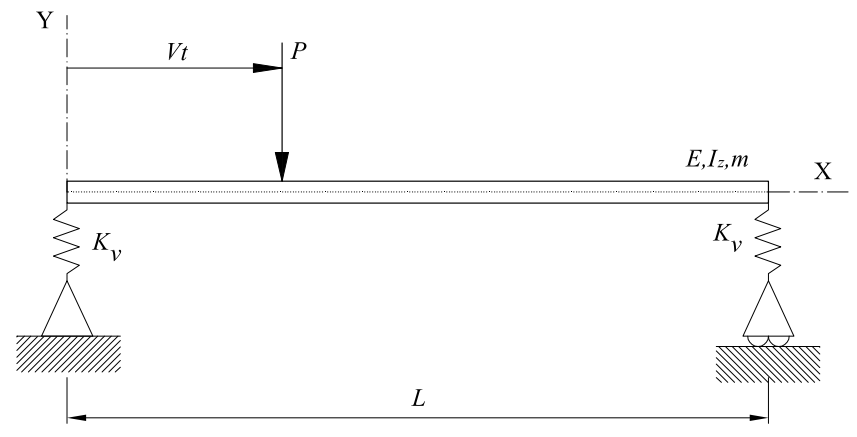

Figure 1: Elastically supported beam traversed by a moving load at constant speed

with $\lambda_{n}=\lambda_{n}(\kappa)$ being the roots of Eq.(11), $\kappa=E I_{z} \pi^{3} /\left(K_{v} L^{3}\right)$ the ratio of the flexural stiffness of the beam to the vertical stiffness of the elastic bearings, where $L, K_{v}$ and $E I_{z}$ are the beam length, vertical stiffness of the support and bending stiffness of the cross section, respectively. The circular frequencies are then defined as follows,

$$
\omega_{n}=\left(\frac{\lambda_{n}}{L}\right)^{2} \sqrt{\frac{E I_{z}}{m}}
$$

with $m$ being the linear mass of the beam. In the S-S case, $\kappa=0$ and $\lambda_{n}=n \pi$, leading to the well-known natural frequencies and mode shapes of this particular case. For the E-S beam, the analytical expressions of the eigenforms are

$$
\phi_{n}(\kappa, l)=\frac{\psi_{n}(\kappa, l)}{\max \left|\psi_{n}(\kappa, l)\right|}
$$

where $l=x / L$ and $\psi_{n}(\kappa, l)$ is defined as,

$$
\begin{gathered}
\psi_{n}(\kappa, l)=\sin \left(\lambda_{n} l\right)+\sinh \left(\lambda_{n} l\right) \frac{\sin \left(\lambda_{n}\right)}{\sinh \left(\lambda_{n}\right)} \\
+\gamma_{1 n}\left(\cos \left(\lambda_{n} l\right)+\cosh \left(\lambda_{n} l\right)+\gamma_{2 n} \sinh \left(\lambda_{n} l\right)\right), 0 \leq l \leq 1 ;
\end{gathered}
$$

$$
\begin{array}{r}
\gamma_{1 n}=\frac{\sinh \left(\lambda_{n}\right)-\sin \left(\lambda_{n}\right)}{\frac{2}{\kappa}\left(\frac{\pi}{\lambda_{n}}\right)^{3} \sinh \left(\lambda_{n}\right)+\cos \left(\lambda_{n}\right)-\cosh \left(\lambda_{n}\right)} \\
\gamma_{2 n}=\frac{\cos \left(\lambda_{n}\right)-\cosh \left(\lambda_{n}\right)}{\sinh \left(\lambda_{n}\right)}
\end{array}
$$

The phenomena of resonance and cancellation are both associated to the free vibrations created by each of the axle loads that have crossed the structure. Such free vibrations 
can possibly accumulate, thus leading to the resonance phenomenon, or vanish at certain speeds of circulation, which corresponds to the cancellation phenomenon previously anticipated by Yang et al. ([17] and [9]), Savin [18] and Pesterev [19]. The amplitude of the $n$th modal response of the beam after the passage of a single moving load $P$ at constant speed $V$ in the so-called free vibration phase $(t>L / V)$ was derived in [14] using the analytical mode shapes of the beam. It can be expressed as

$$
A_{n}=\sqrt{\frac{\dot{\xi}_{n}(t=L / V)^{2}}{\omega_{n}^{2}}+\xi_{n}(t=L / V)^{2}} ;
$$

where $\xi_{n}(t=L / V)$ and $\dot{\xi}_{n}(t=L / V)$ are the initial conditions for the free vibration phase which correspond, respectively, to the $n$th modal deflection of the beam and its derivative at $t=L / V$, during the forced vibration phase, $0 \leq t \leq L / V$. Analytical expressions of these quantities are derived as per,

$$
\begin{array}{r}
\xi_{n}(t=L / V)=\frac{-\xi_{n, s t}}{\max \left|\psi_{n}(\kappa, l)\right|} \\
\cdot\left[\frac{K_{n} \sin \left(\frac{\lambda_{n}}{K_{n}}\right)-\sin \left(\lambda_{n}\right)}{K_{n}^{2}-1}+\left(\frac{\sin \left(\lambda_{n}\right)}{\sinh \left(\lambda_{n}\right)}+\gamma_{1 n} \gamma_{2 n}\right)\right. \\
\cdot \frac{\sinh \left(\lambda_{n}\right)-K_{n} \sin \left(\frac{\lambda_{n}}{K_{n}}\right)}{K_{n}^{2}+1}+\gamma_{1 n} \frac{\cos \left(\frac{\lambda_{n}}{K_{n}}\right)-\cos \left(\lambda_{1 n}\right)}{K_{n}^{2}-1} \\
\left.+\gamma_{1 n} \frac{\cosh \left(\lambda_{n}\right)-\cos \left(\frac{\lambda_{n}}{K_{n}}\right)}{K_{n}^{2}+1}\right], \\
\dot{\xi_{n}(t}=\frac{L / V)=\frac{-\xi_{n, s t} \omega_{n}}{\max \left|\psi_{n}(\kappa, l)\right|}}{\cdot \frac{K_{n} \cos \left(\frac{\lambda_{n}}{K_{n}}\right)-K_{n} \cos \left(\lambda_{n}\right)}{K_{n}^{2}-1}+\left(\frac{\sin \left(\lambda_{n}\right)}{\sinh \left(\lambda_{n}\right)}+\gamma_{1 n} \gamma_{2 n}\right)} \\
\frac{K_{n} \cosh \left(\lambda_{n}\right)-K_{n} \cos \left(\frac{\lambda_{n}}{K_{n}}\right)}{K_{n}^{2}+1}-\gamma_{1 n} \frac{\sin \left(\frac{\lambda_{n}}{K_{n}}\right)-K_{n} \sin \left(\lambda_{1 n}\right)}{K_{n}^{2}-1} \\
+\gamma_{1 n} \frac{K_{n} \sinh \left(\lambda_{n}\right)+\sin \left(\frac{\lambda_{n}}{K_{n}}\right)}{K_{n}^{2}+1},
\end{array}
$$

In the previous expressions $\xi_{n, s t}$ is the static solution of the beam,

$$
\xi_{n, s t}=\frac{P}{M_{n} \omega_{n}^{2}}=\frac{P}{E I_{z} / L^{3}} \cdot \frac{1}{\hat{\Psi}_{n} \lambda_{n}^{4}}
$$

with $M_{n}$ being the nth modal mass in the case of unitary amplitude normalisation, and $\hat{\Psi}_{n}$ is defined as follows,

$$
\hat{\Psi}_{n}=\int_{0}^{1} \phi_{n}^{2}(\kappa, l) d l .
$$

As can be observed Eqs. (6a) and (6b) are only dependent on the parameters $\kappa$, the flexural stiffness of the beam $E I_{z}$ and its length $L$, the constant load $P$ and the nondimensional speed $K_{n}$, which is the quotient between the excitation frequency and the $n$th circular frequency of the beam,

$$
K_{n}=\frac{\lambda_{n} V}{\omega_{n} L}
$$

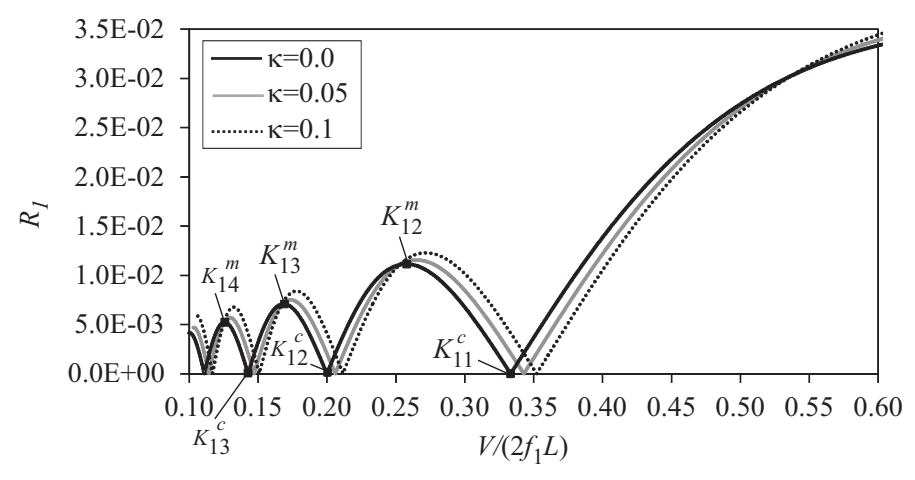

Figure 2: Normalised amplitude of the free vibrations of the first mode in a E-S beam after the circulation of a moving load

In a view to analyse the influence of the supports flexibility $\kappa$ on the amplitude of the free vibration response in a resonance situation, the following normalisation of Eq. (5) is defined,

$R_{n}\left(\kappa, K_{n}\right)=\frac{1}{P /\left(E I_{z} / L^{3}\right)} \sqrt{\frac{\dot{\xi}_{n}(t=L / V)^{2}}{\omega_{n}^{2}}+\xi_{n}(t=L / V)^{2}}$

In Fig. 2 this new expression is plotted for the first flexural mode, $R_{1}$, considering different values of the support flexibility, ranging from $\kappa=0.0$ (rigid supports) to $\kappa=0.1$ (upper limit of the bearings vertical flexibility to be expected in the structures under consideration). Plotting this figure in function of $K_{n}$ can be somewhat mis- 
leading, since $K_{n}$ varies with $\kappa$. Instead, Fig. 2 is plotted versus the nondimensional quotient $V /\left(2 f_{1} L\right)$, standing $f_{1}$ for the frequency of the first flexural mode of the beam. In a resonance situation it is straightforward to verify that $V^{r} /\left(2 f_{1} L\right)=d /(2 j L)$, with $d$ being the so-called characteristic distance between repeated moving loads (which usually identifies a given train set), $j$ the resonance order of the first flexural mode (a positive integer), and $V^{r}$ the resonant speed. Therefore, if we restrict the analysis of the results shown in Fig. 2 to the response of a given beam of span $L$, all the amplitude values with the same nondimensional quotient $V /\left(2 f_{1} L\right)$ can be regarded as corresponding to a resonance phenomenon of order $j$ excited by a vehicle with characteristic distance $d$. In other words, those values represent identical resonance situations generated by a certain convoy, and any difference between them is only caused by the supports flexibility $\kappa$.

As can be seen in Fig. 2 and it was also anticipated by Pesterev et al. [19] for a S-S beam $(\kappa=0.0)$, the amplitude of the free vibrations experiences a number of local maxima which alternate with points of zero amplitude, which correspond to the phenomena of maximum free vibration response and cancellation treated in [14], respectively. Furthermore, this representation reveals an additional behaviour pattern: the curves exhibit higher amplitude values with the increase of the support flexibility $\kappa$ for certain ranges of the quotient $V /\left(2 f_{1} L\right)$; on the contrary, in other regions of the diagram, the amplitude decreases with the increase of $\kappa$. Also, all the free vibration curves for different values of $\kappa$ have a common intersecting point that indicates the change in the mentioned pattern. Although it may be computed numerically, for practical purposes this intersection point will be approximated to the local maxima of the free vibration response of the S-S beam, since both values are very close to each other. Using as a basis the observed evolution of the free vibration response, it is possible to predict whether a certain resonance order $j$ of the first bending mode, due to the passage of a train of characteristic distance $d$, attains a higher amplitude including the vertical stiffness $\kappa$ in the numerical model or does not. This prediction may be obtained from the following expressions:

- The resonance peak response will attain a higher amplitude neglecting the vertical stiffness of the elastic bearings, $\kappa=0.0$, if the quotient $d /(2 j L)$ accomplishes

$$
K_{11}^{c}<\frac{d}{2 j L}<0.53, \text { or } K_{1 i}^{c}<\frac{d}{2 j L}<K_{1 i}^{m} ; i>1 .
$$

- Conversely, the resonance peak response will attain a higher amplitude considering the vertical stiffness of the elastic bearings, $\kappa>0.0$, if the quotient $d /(2 j L)$ accomplishes

$$
K_{1(i+1)}^{m}<\frac{d}{2 j L}<K_{1 i}^{c} ; i \geq 1
$$

The values $K_{1 i}^{m}$ and $K_{1 i}^{c}$ are, respectively, the nondimensional speeds associated to a maximum or a cancellation of the free vibration response of the first mode for the S-S beam, depicted in Fig. 2, Subscript $i$ is a positive integer that designates the $i$ th local maximum or cancellation of the free vibration response, corresponding $i=1$ to the highest nondimensional speed. It should be emphasised that in the S-S case, $K_{1}=V /\left(2 f_{1} L\right)$. These critical points may be numerically sought; the ones related to the highest speeds are shown in table 1

\begin{tabular}{|c||c|c|c|c|}
\hline & $i=1$ & $i=2$ & $i=3$ & $i=4$ \\
\hline$K_{1 i}^{m}$ & 0.7314 & 0.2576 & 0.1687 & 0.1258 \\
\hline \hline & $i=1$ & $i=2$ & $i=3$ & $i=4$ \\
\hline$K_{1 i}^{c}$ & $1 / 3$ & $1 / 5$ & $1 / 7$ & $1 / 9$ \\
\hline
\end{tabular}

Table 1: Values of $K_{1}^{m}$ and $K_{1}^{c}$ corresponding to maxima of the free vibrations and cancellations for the first mode of a S-S beam

Eqs. (11) and (12) provide a very useful criteria to verify whether the introduction of the vertical stiffness of the elastomeric bearings in the numerical model guarantees a conservative prediction of the maximum dynamic response in terms of vertical accelerations or does not. The effectiveness of these expressions will be shown in subsection 5.4 . 


\section{Definition of a representative ensemble of rail-} way bridges for increased traffic speed

Among the different bridge typologies traditionally used for covering the span lengths between $10 \mathrm{~m}$ and $25 \mathrm{~m}$ in conventional lines, some of the most typical ones found in the Spanish railway network have been selected for this study: reinforced concrete slabs and pre-stressed concrete girder decks. The characteristics of the decks analysed in this study are derived from existing structures so as to constitute realistic examples leading to applicable results and conclusions.

Slab bridges are very common in urban surroundings for spans shorter than approximately $15 \mathrm{~m}$, and with a slenderness ratio (depth/span) between 1/12-1/14. Three representative structures have been dimensioned for this study, based on the use of a thick slab which covers a short span with a straightforward, simply supported solution. Their cross section is shown in Fig. 3, where the parameter $h$ is given in table 2 The span lengths are equal to 10, 12.5 and $15 \mathrm{~m}$.

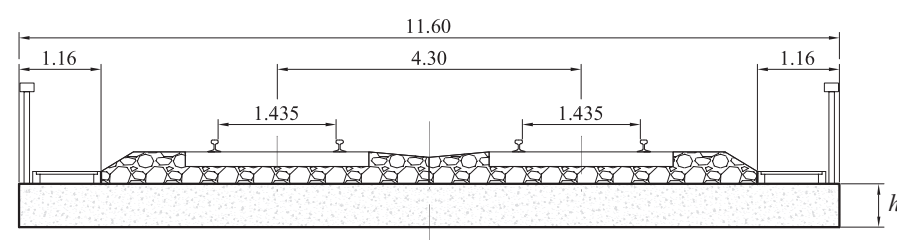

Figure 3: Cross section of reinforced concrete slab bridges under study. Units (m)

As regards the mechanical properties of the bridges (shown in table 2), the nominal value for the concrete strength $f_{c k}$ assumed for the decks is $35 \mathrm{MPa}$. In order to take into account the degradation of material properties due to cracking, which is a frequent phenomenon in this type of decks, the three different Elastic Modules shown in table 3 have been considered in the dynamic analyses. The linear mass values $m$ account for the slab selfweight and also the dead loads of the deck (ballast, rails, sleepers); three different values are considered due to the un-

\begin{tabular}{|c|c|c|c|c|}
\hline & \multirow{3}{*}{$\begin{array}{c}\text { S10 } \\
10 \\
\end{array}$} & \multirow{3}{*}{\begin{tabular}{|c|}
$\mathrm{S} 12.5$ \\
12.5 \\
\end{tabular}} & \multirow{3}{*}{$\frac{\mathrm{S} 15}{15}$} \\
\hline & & & & \\
\hline \multirow{4}{*}{ Slab } & $L(\mathrm{~m})$ & & & \\
\hline & $B(\mathrm{~m})$ & \multicolumn{3}{|c|}{11.6} \\
\hline & $h(\mathrm{~m})$ & 0.77 & 0.96 & 1.15 \\
\hline & $f_{c k}(\mathrm{MPa})$ & \multicolumn{3}{|c|}{35 (table 3) } \\
\hline \multirow{3}{*}{$\begin{array}{l}\text { Track } \\
\text { plattform }\end{array}$} & No.tracks, width & \multicolumn{3}{|c|}{$2, \mathrm{UIC}$} \\
\hline & Rail type & \multicolumn{3}{|c|}{ UIC-60 } \\
\hline & Track eccentricity & \multicolumn{3}{|c|}{2.15} \\
\hline \multirow{3}{*}{ Mass } & $m_{\text {inf }}(\mathrm{kg} / \mathrm{m})$ & 28796 & 34306 & 39816 \\
\hline & $m_{\text {nom }}(\mathrm{kg} / \mathrm{m})$ & 31301 & 36811 & 42321 \\
\hline & $m_{\text {sup }}(\mathrm{kg} / \mathrm{m})$ & 33807 & 39317 & 44827 \\
\hline Supports & Flexibility $\kappa$ & \multicolumn{3}{|c|}{$0,0.05,0.1$} \\
\hline
\end{tabular}

Table 2: Main properties of the concrete slab bridges of lengths $L=[10,12.5,15] \mathrm{m}$

certainty in the determination of the ballast mass and its great contribution to the total weight of the deck. These have been obtained considering variations of $+/-30 \%$ with respect to a nominal value of the ballast layer thickness $\left(h_{n o m}=0.5 \mathrm{~m}\right)$. The vertical stiffness of the elastomeric bearings is given in table 2 in terms of $\kappa$ : three values per bridge geometry have been considered, ranging from $\kappa=0.0$ (simply supported deck) to $\kappa=0.1$. Taking into account the mass variations, the different supports flexibility for each deck, as well as the variations of the Elastic Modulus, a total number of 81 case studies are analised for this typology. The data columns of table 2 include a heading with the nomenclature adopted to identify each case study. Also, a similar designation is used in table 4 for the girder bridges.

\begin{tabular}{|l||l|l|}
\hline \multirow{2}{*}{$f_{c k}=35 \mathrm{MPa}$} & $E_{\text {nom }}(\mathrm{Pa})$ & $3.4849 \cdot 10^{10}$ \\
\cline { 2 - 3 } & $E_{-10 \%}(\mathrm{~Pa})$ & $3.1365 \cdot 10^{10}$ \\
\cline { 2 - 3 } & $E_{-20 \%}(\mathrm{~Pa})$ & $2.7879 \cdot 10^{10}$ \\
\hline
\end{tabular}

Table 3: Values of the Elastic Modulus considered for the slab bridges

At present, solutions based on pre-stressed concrete girder bridges are less usual in high-speed lines, since they 


\begin{tabular}{|c|c|c|c|c|c|c|c|c|}
\hline & & GB10 & GB12.5 & GB15 & GB17.5 & GB20 & GB22.5 & GB25 \\
\hline \multirow{5}{*}{ Slab } & $L(\mathrm{~m})$ & 10 & 12.5 & 15 & 17.5 & 20 & 22.5 & 25 \\
\hline & $B(\mathrm{~m})$ & \multicolumn{7}{|c|}{11.6} \\
\hline & $h(\mathrm{~m})$ & 0.22 & 0.22 & 0.25 & 0.25 & 0.25 & 0.25 & 0.25 \\
\hline & $\rho\left(\mathrm{kg} / \mathrm{m}^{3}\right)$ & \multicolumn{7}{|c|}{2500} \\
\hline & $f_{c k}(\mathrm{MPa})$ & \multicolumn{7}{|c|}{30} \\
\hline \multirow{8}{*}{ Girders } & $N_{\text {girder }}, d_{\text {girder }}$ & $6,2.0$ & $6,2.0$ & $6,2.0$ & $6,2.0$ & $5,2.275$ & $5,2.275$ & $5,2.275$ \\
\hline & $I_{h}\left(\mathrm{~m}^{4}\right)$ & 0.011 & 0.0228 & 0.0396 & 0.062 & 0.1117 & 0.1599 & 0.2181 \\
\hline & $I_{v}\left(\mathrm{~m}^{4}\right)$ & 0.0085 & 0.0085 & 0.0085 & 0.009 & 0.0202 & 0.0202 & 0.0203 \\
\hline & $J\left(\mathrm{~m}^{4}\right)$ & 0.0017 & 0.0018 & 0.0019 & 0.002 & 0.0078 & 0.0081 & 0.0083 \\
\hline & $A\left(\mathrm{~m}^{2}\right)$ & 0.2276 & 0.2516 & 0.2756 & 0.299 & 0.4787 & 0.5087 & 0.5387 \\
\hline & $h_{\text {girder }}(\mathrm{m})$ & 0.6 & 0.8 & 1.0 & 1.2 & 1.3 & 1.5 & 1.7 \\
\hline & $\rho\left(\mathrm{kg} / \mathrm{m}^{3}\right)$ & \multicolumn{7}{|c|}{2500} \\
\hline & $f_{c k}(\mathrm{MPa})$ & \multicolumn{7}{|c|}{50} \\
\hline \multirow{3}{*}{$\begin{array}{l}\text { Track } \\
\text { plattform }\end{array}$} & No.tracks, width & \multicolumn{7}{|c|}{$2, \mathrm{UIC}$} \\
\hline & Rail type & \multicolumn{7}{|c|}{ UIC-60 } \\
\hline & Track eccentricity & \multicolumn{7}{|c|}{2.15} \\
\hline \multirow{3}{*}{ Mass } & $m_{\text {inf }}(\mathrm{kg} / \mathrm{m})$ & 16260 & 16620 & 17850 & 18210 & 19699 & 20075 & 20450 \\
\hline & $m_{\text {nom }}(\mathrm{kg} / \mathrm{m})$ & 18765 & 19125 & 20355 & 20715 & 22205 & 22581 & 22956 \\
\hline & $m_{\text {sup }}(\mathrm{kg} / \mathrm{m})$ & 21271 & 21631 & 22861 & 23221 & 24711 & 25086 & 25461 \\
\hline Supports & Flexibility $\kappa$ & \multicolumn{7}{|c|}{$0,0.05,0.1$} \\
\hline
\end{tabular}

Table 4: Main properties of the girder bridges of span lengths ranging from 10 to $25 \mathrm{~m}$.

exhibit lower resistance to torsion when compared to other solutions. Despite this fact, a number of girder bridges were specifically built in the late 80 's for the first highspeed railway line in Spain (Madrid-Sevilla), and they are also very common in conventional lines which, according to current trends, often experience increases of the operating train speed. This typology usually covers span lengths between $10 \mathrm{~m}$ and $25 \mathrm{~m}$, with slenderness ratios no higher than 1/13. Fig. 4 and table 4 summarise the geometry and main properties of a series of prestressed concrete girder bridges that have been dimensioned for this study. Considering the three different variations of linear mass for each bridge geometry, due to the uncertainties in the determination of the ballast layer thickness, and also the three different supports flexibility for each deck, a total number 63 pre-stressed concrete girder bridge models are analysed.

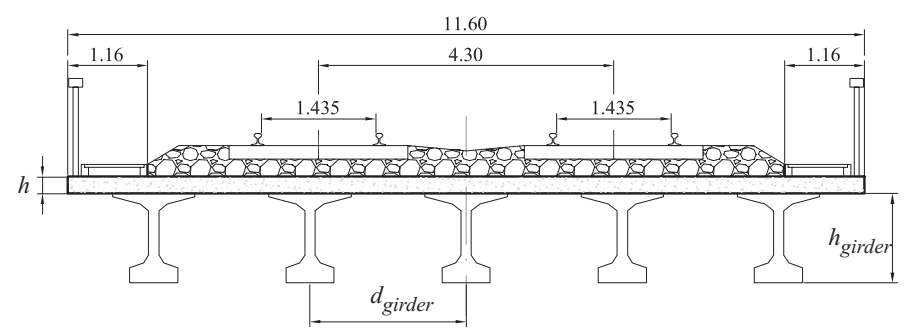

Figure 4: Cross section of pre-stressed concrete girder bridges under study. Units (m) 


\section{Numerical analysis}

\subsection{Finite element model}

The dynamic performance of the bridges under railway traffic has been predicted using a finite element (FE) model implemented in a Fortran code (Fig. [5), whose main features are the following: (i) the deck behaviour is simulated by means of an orthotropic thin plate discretised in linear varying curvature FEs, which are $C^{1}$ compatible triangular elements with 12 degrees of freedom as shown in Figure 5 (Felippa [20]); (ii) the laminated rubber bearings of the deck girders are included in the model as an equivalent vertical stiffness uniformly distributed along the abutments; (iii) different mass density elements are used in order to concentrate the weight of the ballast, sleepers and rails over the central portion of the plate; (iv) a point load model is adopted for the railway excitation, therefore neglecting vehicle-structure interaction effects; these loads move along the rails of one of the tracks, with eccentricities of $1.4325 \mathrm{~m}$ and $2.8675 \mathrm{~m}$; (v) the dynamic equations of motion are transformed into modal space and numerically integrated applying the Newmark- $\beta$ linear acceleration algorithm, taking into account a proper number of modes; (vi) uniform damping ratios are assigned to all mode contributions, in accordance to the minimum values recommended by EC1, which are $1 \%$ for spans $L \geq 20 \mathrm{~m}$ and $1+0.07(20-L)$ in the other cases.

The model main features can be easily implemented using a commercial software and are in accordance with the European Standards. For these reasons, this model could be a suitable simplification for the verification of the Serviceability Limit State of vertical acceleration in practical applications. The effect of track irregularities are considered with the multiplication of the dynamic results by the factor $\left(1+\varphi^{\prime \prime} / 2\right)$ defined in EC1.

As the track rigidity has not been included in the model, when a load enters or exits the bridge crossing a border element a transient phenomenon takes place due to the

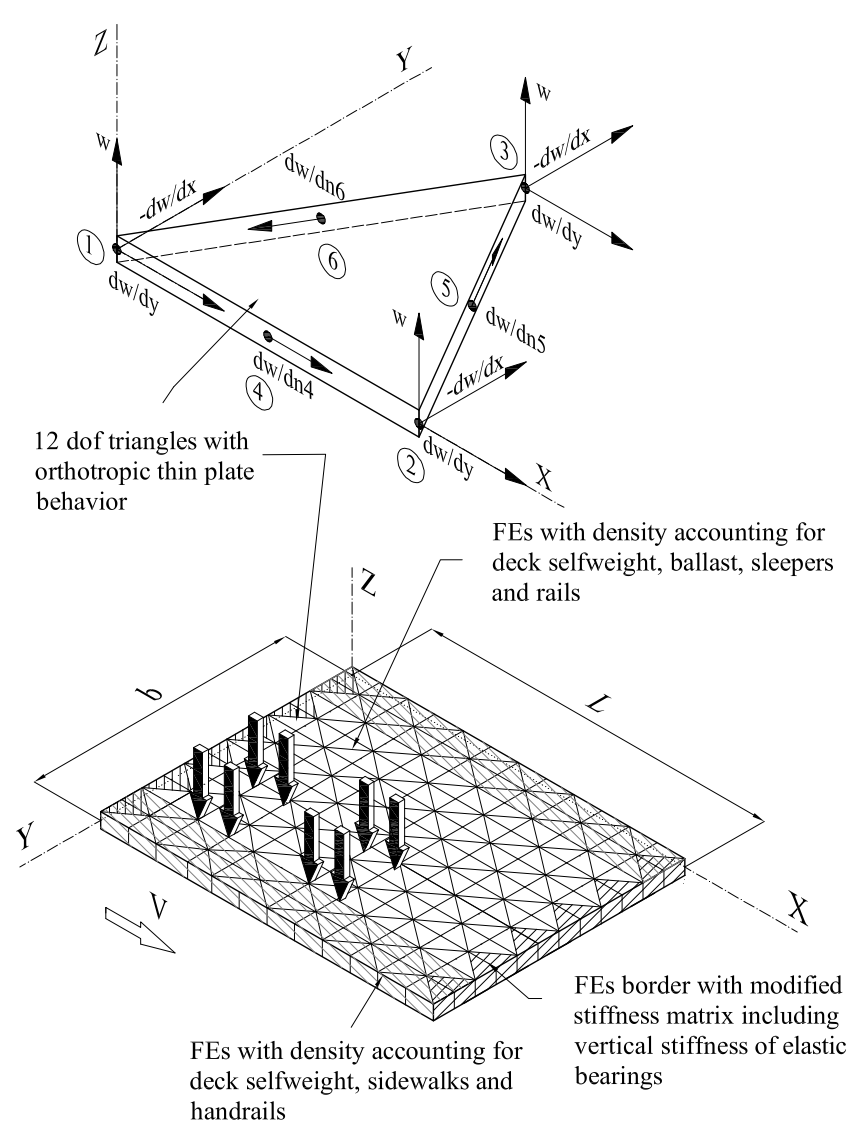

Figure 5: Orthotropic plate FE model 
presence of the elastic bearings which leads to unrealistic high-frequency modal contributions of the plate [21]. This numerical problem has been solved including the distributive effect of rails, sleepers and ballast during the application process of the wheel loads when they are close to the abutments. To this end, each axle load is distributed throughout a load-print which is based on the ZimmermanTimoshenko solution for an infinite beam on Winkler foundation, as described in [21].

The computation of the orthotropic plate constants, $D_{x}, D_{y}, D_{1}, D_{2}, D_{x y}$ and $D_{y x}$, from the real bridge deck mechanical properties is not included in this document for the sake of brevity, but it is explained with detail in literature (see [22, 23]).

\subsection{Description of the analysis procedure}

The vertical response in terms of accelerations of the double-track decks is computed in the time domain at 25 points of study equally spaced over the track platform (Fig. 6), and under the circulation of the ten HSLM-A trains defined in EC1 in a range of velocities between 100 and $420 \mathrm{~km} / \mathrm{h}$ in $1.80 \mathrm{~km} / \mathrm{h}$ steps. As can be seen in Fig. 6 , the vertical accelerations are computed at five sections (A,B,C,D ,E) corresponding to $\mathrm{x} / L=(0,0.25,0.5,0.75,1)$, with $L$ being the span length. For each section, five postprocessing points $(1,2,3,4,5)$ are considered corresponding to $\mathrm{y} / B=(0.1,0.3,0.5,0.7,0.9)$, with $B$ being the span width

Three analyses per bridge deck model have been performed, accounting for a different number of mode contributions which are (i) all modes with frequencies up to the maximum limit prescribed by EC; (ii) only the longitudinal bending modes among those satisfying the preceding criterion (i); and (iii) the first two mode shapes, which correspond to the first longitudinal bending and first torsion modes of the decks in all cases. The comparison of (i), (ii) and (iii) will provide valuable insight into the relative influence of the different modal contributions, focusing

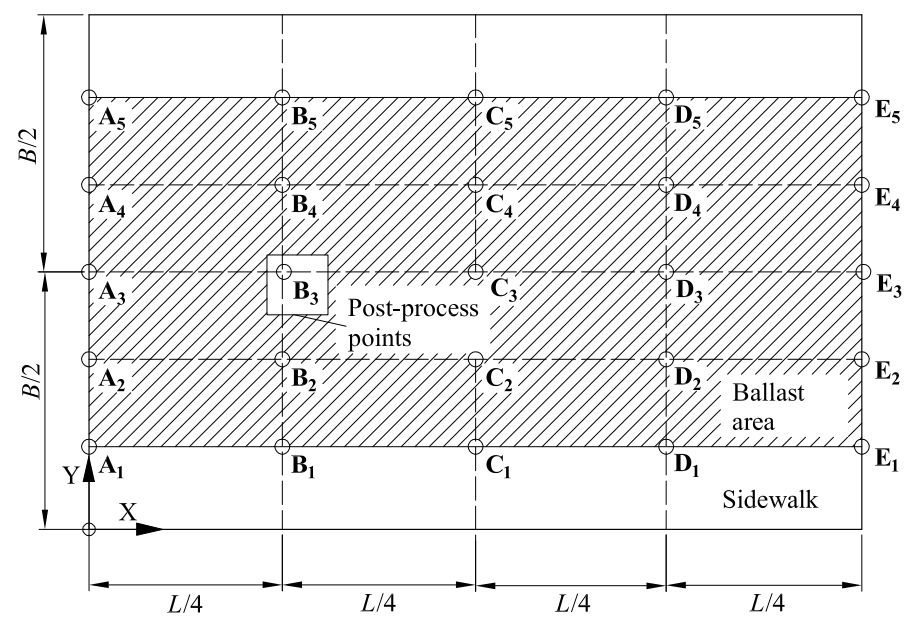

Figure 6: Location of post-process points

particularly in the first two eigenforms, as well as on the additional effects due to higher modes up to $30 \mathrm{~Hz}$.

Envelopes of maximum acceleration in the deck platform have been computed for each numerical model and speed of circulation, and have been grouped in terms of (i) the bridge span and typology, (ii) the number of mode contributions accounted for, and (iii) the value of the vertical stiffness of the elastic bearings.

Table 5 gathers the nomenclature adopted for the identification of these dynamic envelopes, where the initials XX stand for the bridge geometry designation that was firstly introduced in tables 2 and 4 (e.g. slab bridge of $10 \mathrm{~m}$ span length, S10; girder bridge of $25 \mathrm{~m}$ span length, GB25).

\section{Results}

Due to the large amount of case studies analysed in this work, for the sake of conciseness only a summary of the most representative results is presented, along with the main conclusions derived.

\subsection{Summary of natural frequencies and mode shapes}

In Fig. 7 the fundamental frequency $f_{1}$ of all the bridges of study, which corresponds to the first longitudinal bending mode in all cases, is presented as a function of the span length. All these values are enclosed by the 


\begin{tabular}{|l||c|c|c|}
\cline { 2 - 4 } \multicolumn{1}{l||}{} & long. bending & 1st bending +1 st torsion & EC frequencies \\
\hline$\kappa=0$ & XX.SS.b & XX.SS.b+t & XX.SS.all \\
\hline$\kappa=0.05$ & XX.ES(0.05).b & XX.ES(0.05).b+t & XX.ES(0.05).all \\
\hline$\kappa=0.1$ & XX.ES(0.1).b & XX.ES(0.1).b+t & XX.ES(0.1).all \\
\hline
\end{tabular}

Table 5: Nomenclature adopted for the analyses performed

frequency band of EC, which entails that at speeds lower than $200 \mathrm{~km} / \mathrm{h}$ significant vibrations are not expected.

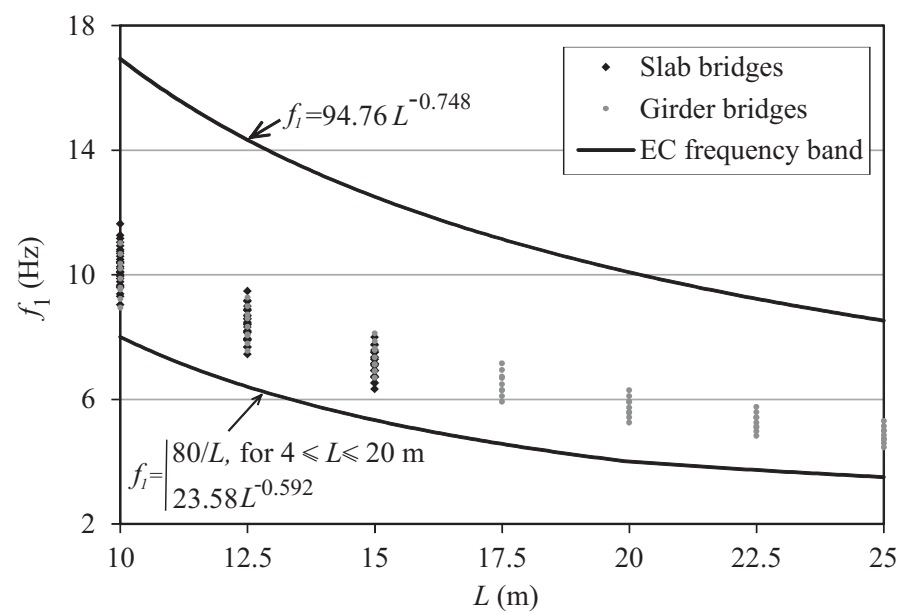

Figure 7: First natural frequency of the bridges of study versus their span length

The second lowest natural frequency, $f_{2}$, is associated to the first torsion mode in all case studies. Table 6 shows the quotients $f_{2} / f_{1}$ predicted in the bridges, where the shaded cells highlight the bridge types that could be modelled as beams according to EC1.

The variations of $E$ assumed for the slab bridges have no influence in the ratios shown in table 6. As can be observed the span length and the vertical stiffness of the elastic bearings have little influence in the quotient $f_{2} / f_{1}$ for the girder bridges. Conversely, in the slab bridges the frequency ratio $f_{2} / f_{1}$ raises with the span length and diminishes at any increase of the flexibility $\kappa$ of the elastic supports.

For frequencies higher than $f_{2}$, eigenforms of transverse bending, torsion or longitudinal bending appear in alternate order. As an example, Fig. 8 shows the mode shapes and natural frequencies below $30 \mathrm{~Hz}$ of the girder bridge

\begin{tabular}{|c|c|c|c|c|c|}
\hline \multicolumn{2}{|c|}{$f_{2} / f_{1}$} & S10 & $\mathrm{S} 12.5$ & $\mathrm{~S} 15$ & GB10-GB25 \\
\hline \multirow{3}{*}{$\kappa=0.0$} & $m_{\text {inf }}$ & 1.60 & 1.84 & 2.11 & $1.08-1.09$ \\
\hline & $m_{n o m}$ & 1.62 & 1.86 & 2.13 & $1.10-1.11$ \\
\hline & $m_{\text {sup }}$ & 1.63 & 1.88 & 2.14 & $1.11-1.12$ \\
\hline \multirow{3}{*}{$\kappa=0.05$} & $m_{i n f}$ & 1.51 & 1.7 & 1.89 & 1.08 \\
\hline & $m_{n o m}$ & 1.53 & 1.71 & 1.90 & 1.10 \\
\hline & $m_{\text {sup }}$ & 1.55 & 1.73 & 1.92 & $1.11-1.12$ \\
\hline \multirow{3}{*}{$\kappa=0.1$} & $m_{\text {inf }}$ & 1.46 & 1.61 & 1.75 & $1.07-1.08$ \\
\hline & $m_{n o m}$ & 1.47 & 1.62 & 1.77 & $1.09-1.10$ \\
\hline & $m_{\text {sup }}$ & 1.49 & 1.64 & 1.79 & $1.11-1.12$ \\
\hline
\end{tabular}

Table 6: Frequency ratios $f_{2} / f_{1}$ in all the bridges of study

of $25 \mathrm{~m}$ span, GB25, with mass $M_{t a b, s u p}$ and considering $\kappa=0.0$. When the vertical stiffness of the elastic supports is included in the numerical model a certain reduction of natural frequencies and also variations in the curvature of the eigenforms, specially along the abutments lines, are noticeable, being the modes of higher frequencies the most affected ones. However no significant changes in the order of appearance of the different eigenforms is observed in the range of the supports flexibility considered $\kappa=[0,0.1]$.

\subsection{Maximum acceleration envelopes}

Fig. 9 shows the envelopes of the maximum acceleration for all the case studies versus the circulating speed. In these plots each curve represents the envelope of maximum acceleration of all the bridges with the same span, typology and $\kappa$ value, under the passage of HSLM-A trains. These results have been computed including all the mode contributions prescribed by EC, and have been multiplied by the track irregularity factor $\left(1+\varphi^{\prime \prime} / 2\right)$ according to EC1. 

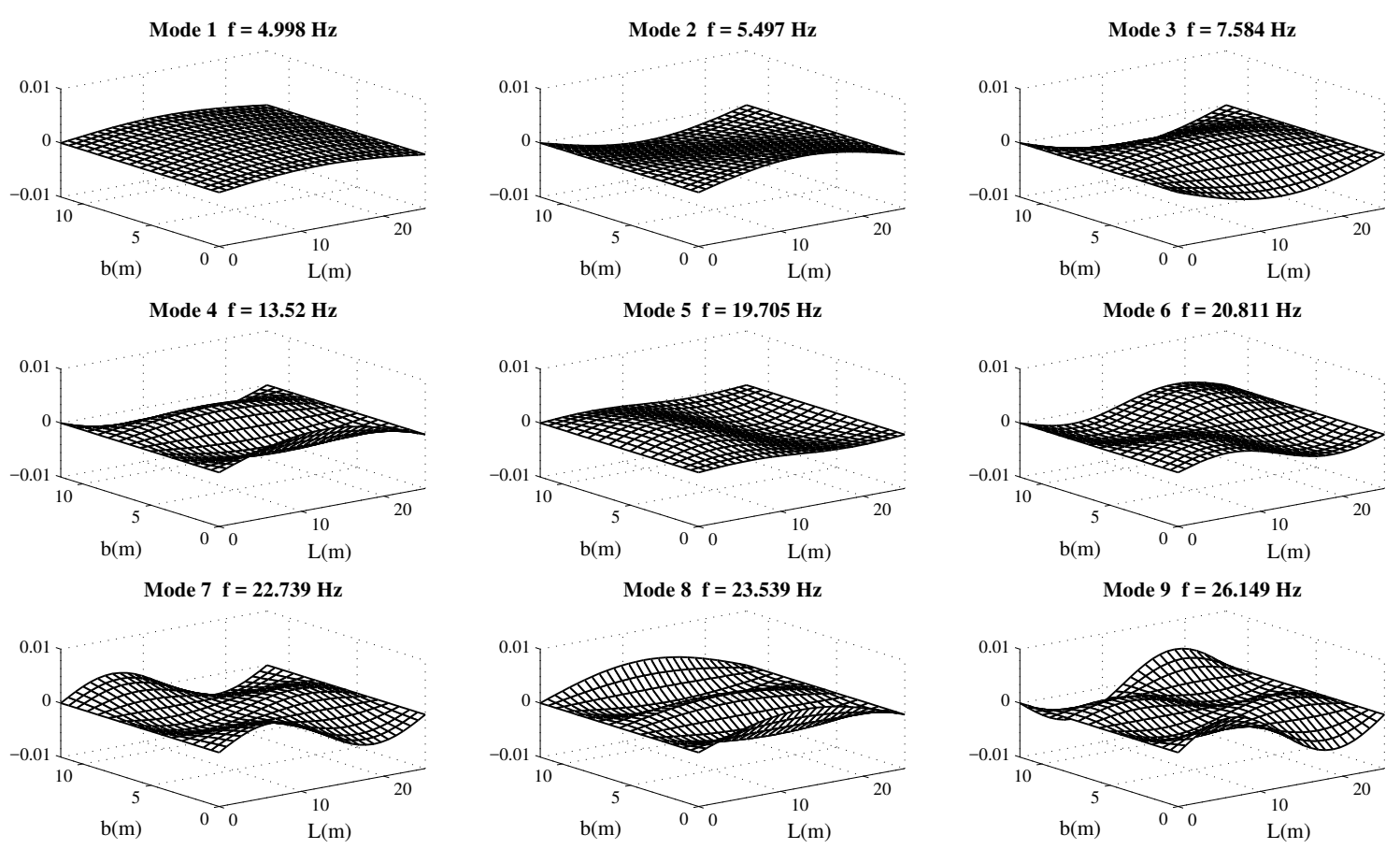

Figure 8: Frequencies and eigenforms below $30 \mathrm{~Hz}$ of the girder bridge GB25 of mass $m_{n o m}$ considering $\kappa=0.0$

Vertical lines of the same colour as the acceleration envelopes indicate the maximum admissible circulating speeds which keep the acceleration levels below admissible limits. These speed values have been obtained considering that accelerations 15\% higher than the Serviceability Limit of $3.5 \mathrm{~m} / \mathrm{s}^{2}$ may still be acceptable, since this limit is affected by a safety factor of 2 .

As can be seen the maximum acceleration values attained in the slab bridges (Figs. 9(c) and (d)) are much lower than the ones corresponding to the girder bridges of the same length (Figs. 9(a) and (b)). This fact is related to the lower mass of the latter typology and also to the higher values of $f_{1}$ associated to the slab bridges. At maximum speeds up to $270 \mathrm{~km} / \mathrm{h}$ slab bridges may still exhibit an acceptable behaviour in terms of vertical accelerations, whereas the girder bridges may undergo inadmissible acceleration levels at lower speeds, close to $200 \mathrm{~km} / \mathrm{h}$. This latter fact largely justifies why girder bridges are rarely found in the high speed lines of more recent construction.

The increase of span length has, in general, a beneficial effect in the maximum vibration levels attained at the highest speeds, due to the increment of mass. However, as was derived from Fig. 2 there are other factors (e.g., $d / L$ ratio, $\kappa)$ that vary the pattern of resonances, and also the resonance speeds decrease with the span length and $\kappa$. These effects may mitigate the favourable influence of the span length. For instance, in Fig 9(a) and (b) the span of $17.5 \mathrm{~m}$ exhibits a resonance peak at $408 \mathrm{~km} / \mathrm{h}$ that significantly raises the acceleration levels. Furthermore, at velocities between $200 \mathrm{~km} / \mathrm{h}$ and $280 \mathrm{~km} / \mathrm{h}$, the dynamic behaviour of girder bridges in terms of accelerations does not tend to improve with the span length.

It should also be emphasised that the maximum acceleration levels in all the bridges of study take place at mid span. This points out the predominant contribution of eigenforms with one half-sine wave along the load path, such as the first longitudinal bending mode or the first torsion one.

\subsection{Effect of the contribution of transverse vibration modes \\ 5.3.1. Reinforced concrete slab bridges \\ The dynamic performance of this type of bridge in terms of maximum vertical accelerations is mainly gov- erned by resonances of the first longitudinal bending mode;}



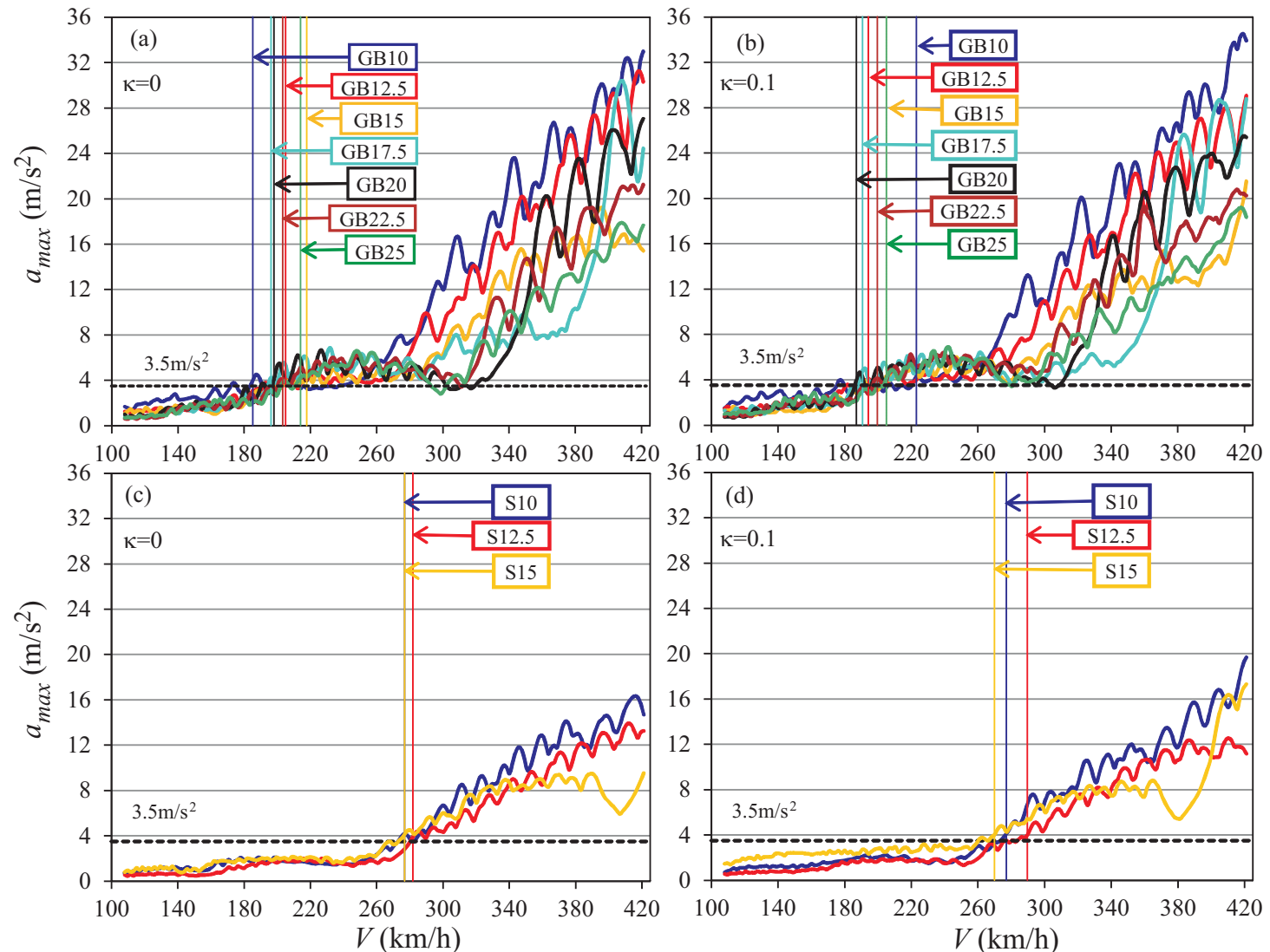

Figure 9: Envelopes of acceleration of the bridges of study
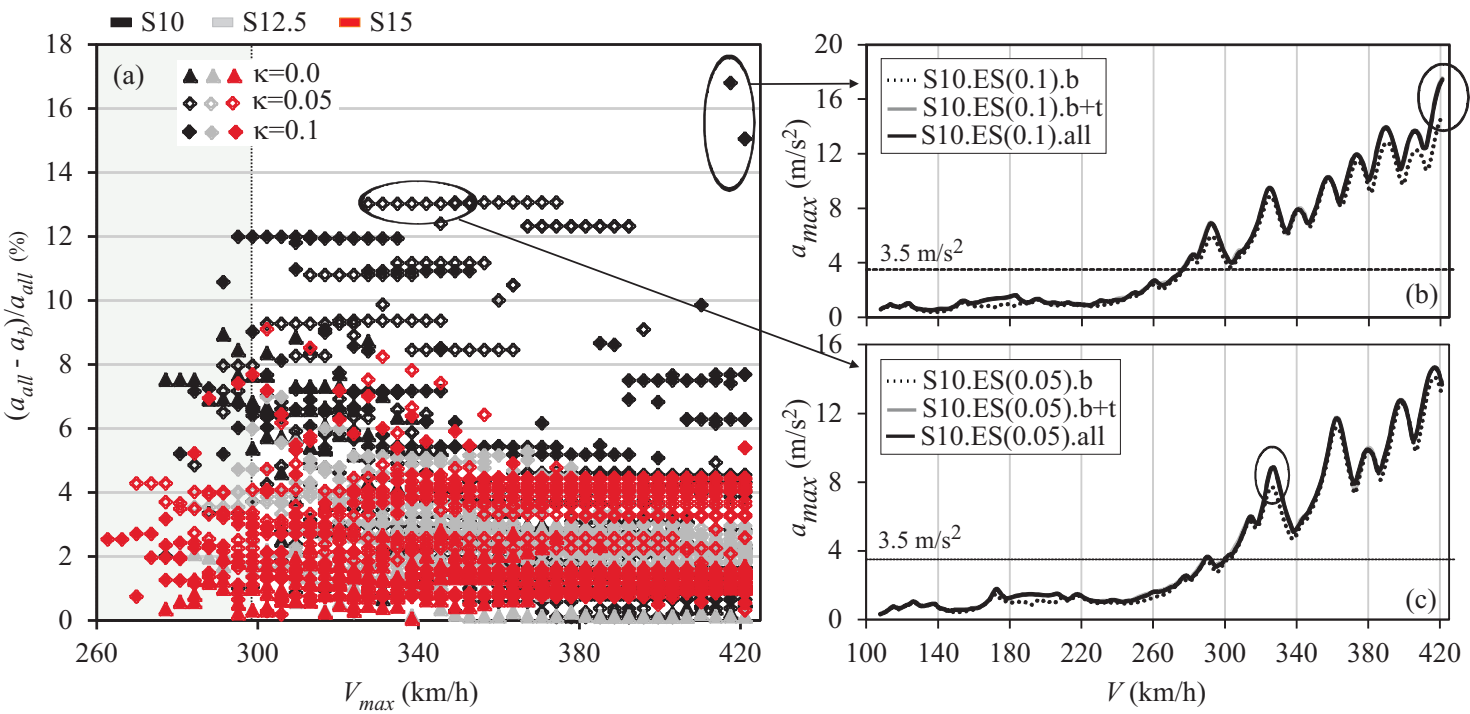

Figure 10: Influence of mode contribution in the prediction of the maximum acceleration levels for slab bridges 
this behaviour is intensified by the increase of the span length and the reduction of the supports flexibility. This can be derived from the observation of Fig. 10, which is explained in what follows.

Fig. 10(a) shows a numerical quantification of the differences between the prediction of the maximum acceleration levels considering only the longitudinal bending modes $\left(a_{b}\right)$ and the accelerations obtained also considering the remaining contributions up to the EC frequency limit $\left(a_{\text {all }}\right)$. Each marker of the plot represents a difference in the prediction of these accelerations, evaluated as $\left(a_{a l l}-a_{b}\right) / a_{a l l} \times 100$.

In this quotient the maximum acceleration for each speed $V_{\max }$ is obtained considering also every lower speed, i.e. is obtained in the range of velocities $\left[100 \mathrm{~km} / \mathrm{h}, V_{\max }\right]$. In such way, only the most significant peaks take part in the evaluation of the relative difference. The speed $V_{\max }$ is indicated in the horizontal axis in steps of $3.6 \mathrm{~km} / \mathrm{h}$. Also differences derived from acceleration values being both of them below $3.5 \mathrm{~m} / \mathrm{s}^{2}$ have been excluded from the plot, since they are not critical for ballast stability.

The shaded area in the range of speeds between 260 $\mathrm{km} / \mathrm{h}$ and $290 \mathrm{~km} / \mathrm{h}$ is specially interesting, since the maximum acceleration levels are slightly beneath or above 3.5 $\mathrm{m} / \mathrm{s}^{2}$ (see Fig. 9) and therefore, significant differences in the prediction of the acceleration levels within this range may be crucial for a safe prediction of the admissible speed limits for this typology. As can be seen the highest differences appear in the elastically supported cases. EC1 only allows to ignore the torsion modes when the supports are rigid, which seems in accordance with these results.

Fig. 10(b) and (c) show the envelopes of vertical acceleration for the two cases in which the highest influence of modes other than longitudinal bending ones has been detected. The results show that the predominance of the first longitudinal bending mode contribution to the maximum response is evident, and becomes even more significant with the increase of the span length.
From the analysis of this typology in terms of the mode contributions affecting the maximum acceleration levels of the deck, the following conclusions can be remarked: (i) the contribution of the first longitudinal bending mode prevails; (ii) modal contributions different from the first longitudinal bending and the first torsion mode have little influence; (iii) the contribution of the first torsion mode may slightly increase the amplitude levels of the resonances of the first vertical bending mode; this effect is more clear in the elastically supported cases. Except in the worst scenario shown in Fig. 10(b), which correspond to the shortest slab bridge and the most flexible supports, the differences between the maximum acceleration computed just with the longitudinal bending modes and with all the modes are kept below $14 \%$.

\subsubsection{Pre-stressed concrete girder bridges}

As previously done for the slab bridges, Fig. [11(a) shows the numerical quantification of the differences in the prediction of the maximum acceleration levels when all the modes are included or only the longitudinal bending ones, evaluated as $\left(a_{a l l}-a_{b}\right) / a_{a l l} \times 100$. Since most of the results fall below $10 \%$ and this difference is not significant to draw practical conclusions, for the sake of clarity the vertical axis is limited to the range 10\%-40\%.

From the analysis of the results it can be derived that the contribution of the first mode to the maximum dynamic response is also relevant, though the contribution of other eigenforms is more noticeable than in the slab bridges, especially in the cases with flexible supports. Mode shapes other than the ones that can be predicted with a simple beam model start to be determinant for spans longer than $15 \mathrm{~m}$, or even for the spans of $12.5 \mathrm{~m}$ when the most flexible elastic supports are considered. This can be seen in Fig. 11(a), where all the differences above $15 \%$ correspond to the longest spans $(\mathrm{L}=[17.525] \mathrm{m})$ and with very flexible supports $(\kappa=0.1)$. In these cases resonances of the first torsion mode increase significantly the maximum 


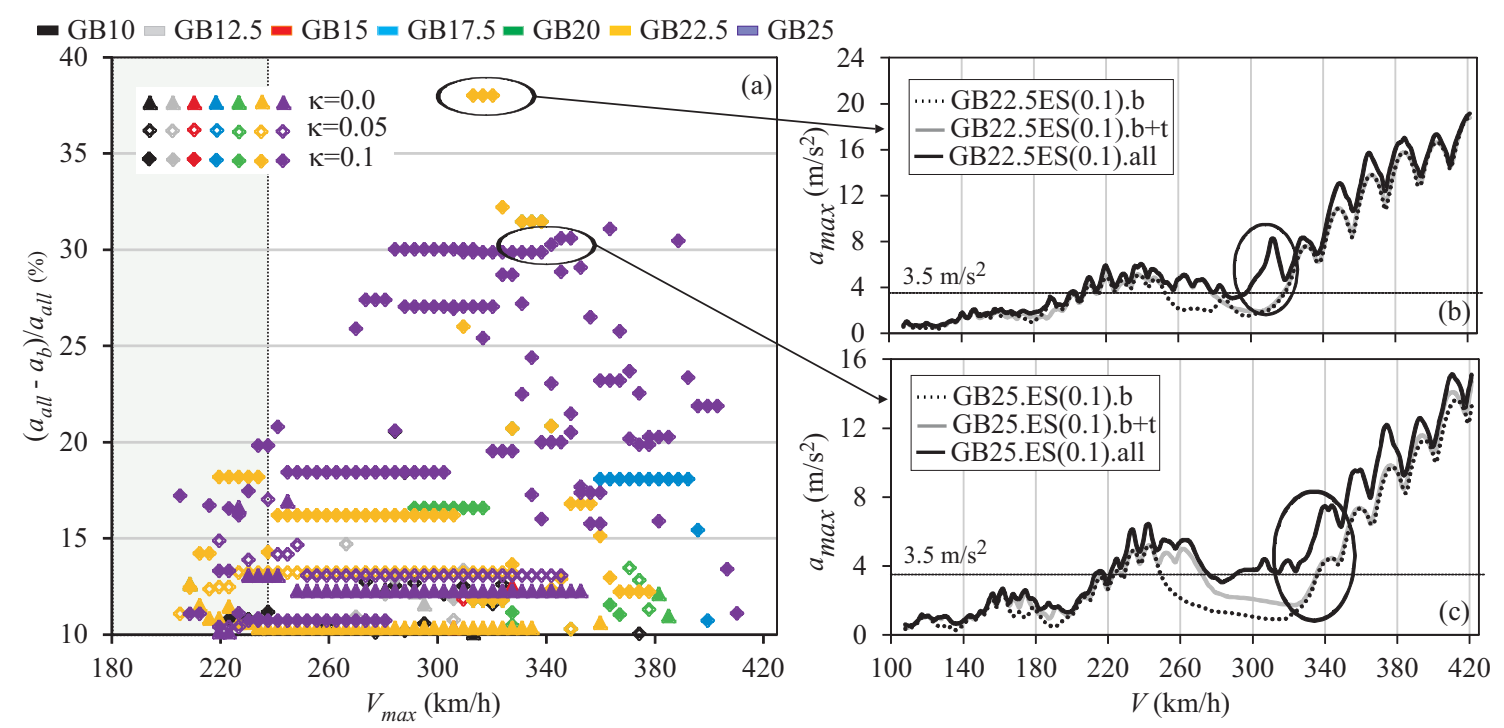

Figure 11: Influence of mode contribution in the prediction of the maximum acceleration levels for girder bridges

level of vibrations. However, the first resonance peaks that lead to inadmissible amplitudes due to the contribution of the torsion mode are usually preceded by resonances of the first longitudinal bending one, occurring at lower speeds, which attain similar or higher amplitudes (see Fig. 11(b) at $260 \mathrm{~km} / \mathrm{h}$ ). The differences in the shaded area of 11(a) are quite low; they are kept below $10 \%$ when the points associated to the longest spans are excluded. As explained below, in the longest spans these higher differences are mainly due to higher frequency modes.

Regarding the contribution of the modes higher than the first torsion one, it has been observed that they have little influence in the acceleration envelopes of the shortest spans. However they may have a significant contribution in certain cases, which correspond to the bridges of the longest spans ( $L=22.5 \mathrm{~m}, L=25 \mathrm{~m})$ on very flexible elastic supports $(\kappa=0.1)$; this is reflected in Fig. 11(a) with the highest differences in the plot. The envelopes of maximum acceleration of the two worst scenarios are shown in Fig. 11(b) and (c); it is important to highlight that this particular behaviour has only been observed in the bridges of $22.5 \mathrm{~m}$ and $25 \mathrm{~m}$ of span length when the most flexible elastic support is considered. As can be seen, despite the influence of higher frequency modes, the response of the bridges still becomes inadmissible due to resonances of the first longitudinal bending mode. Therefore, the prediction of the admissible speed limits that keep the maximum acceleration levels below $3.5 \mathrm{~m} / \mathrm{s}^{2}$ could be also made with a beam-like model for a preliminary assessment.

As a conclusion, three main ideas can be highlighted regarding the dynamic behaviour of the studied girder bridges: (i) the contribution of the first longitudinal bending mode is significant for the prediction of the maximum acceleration levels and suffices for a preliminary assesment; (ii) remarkable resonances of the first torsion mode may appear in the range of speeds of interest, though they are not determinant for the prediction of the maximum admissible speeds of circulation since other resonances of the first longitudinal bending mode occur at lower speeds with comparable amplitudes; (iii) modes higher than the first torsion one contribute to the maximum dynamic response for the longest spans $(L=22.5 \mathrm{~m}, L=25 \mathrm{~m})$ with the most flexible elastic supports $(\kappa=0.1)$.

\subsection{Effect of the vertical stiffness of elastomeric bearings}

The introduction of the vertical stiffness of the elastic supports in the numerical models has two main effects in the prediction of the dynamic behaviour of the bridge: (i) 
reductions of the natural frequencies, and therefore of the critical speeds, and (ii) variations of the resonance amplitudes, which may increase or diminish due to the alterations experienced by the mode shapes, and to the associated variation in the free vibration response of the bridge created by each passing load, as explained in section 2 .

\subsubsection{Reinforced concrete slab bridges}

Regarding the variations of the natural frequencies with the flexibility of the supports, in the slab bridges a maximum reduction of $6 \%$ has been found in the frequency of the first longitudinal bending mode for the elastically supported cases with $\kappa=0.1$, with respect to the rigid supported cases. For the first torsion mode differences up to $22 \%$ have been detected; however, as the first mode prevails in the resonance response, this significant frequency reduction of the first torsion mode is not relevant in terms of maximum acceleration levels. Fig. 12(c) shows an example of the subsequent shift to lower speeds of the resonance peaks with the increase of the supports flexibility. A particular resonance of the first bending mode which is clearly above $3.5 \mathrm{~m} / \mathrm{s}^{2}$ only when the supports are considered as rigid has been marked with vertical arrows.

As regards the influence of $\kappa$ on the amplitude of the resonances, Fig. 12(a) shows another numerical quantification; in this case the maximum acceleration levels considering the highest flexibility of the supports $\left(a_{0.1}\right)$ are compared with the ones obtained with rigid supports $(a)$, using the expression $\left(a_{0.1}-a\right) / a \times 100$. This comparison is made separately for the accelerations predicted with only bending modes, the first bending and torsion modes and all the mode contributions up to the limit prescribed by EC. Also, differences derived from accelerations that are both below $3.5 \mathrm{~m} / \mathrm{s}^{2}$ are excluded from the plot. In this case, in a view to evaluate the sole influence of $\kappa$ in the amplitude of identical resonances, maximum accelerations up to each speed are obtained considering also every lower speed as in Fig. 10(a) and 11(a), but are rather displayed in terms of nondimensional speed $\left[V /\left(2 f_{1} L\right)\right]_{\max }$ as shown in Fig. 12(a).

The vertical dotted trace in Fig. 12(a) indicates the position of $K_{11}^{c}$. As can be seen all the differences are negative and are located above this value, meaning that resonance peaks with associated inadmissible acceleration levels take place at speeds higher than the ones corresponding to the first cancellation of the first longitudinal bending mode. As the contribution of this mode prevails for this type of bridge, according to Eq.(11) the resonance amplitudes should attain higher values if the vertical stiffness of the elastic bearings is neglected. This fact is in accordance with the negative differences shown in Fig. 12(a).

Fig. 12 (b) and (c) show the envelopes of maximum acceleration of the slab bridge with the highest influence of $\kappa$ on the resonance amplitudes; they are plotted versus $V /\left(2 f_{1} L\right)$ and $V$, respectively. As predicted by Eq.

the resonance peaks derived from a numerical model with rigid supports are higher, followed by the values predicted with $\kappa=0.05$ and $\kappa=0.1$.

The reduction of the natural frequencies caused by the flexibility of the supports may lead to the appearance of new resonances at the highest speeds of the range of interest, which are not present in the S-S case. However, the only effect of the reduction of the resonance speeds could be compensated by increasing in $6 \%$ the circulating speeds of the trains for the prediction of the acceleration levels in the S-S model. In this regard, the 1.2 factor affecting the speed that EC1 prescribes also intends to take into account the uncertainty regarding the natural frequencies.

The previous results show that, under important uncertainties in the determination of the vertical stiffness of the elastomeric bearings, a safe and conservative prediction of the maximum acceleration values may be achieved neglecting the flexibility of these elements and assuming them as rigid instead. The supports flexibility may lead to maximum reductions of the resonance amplitudes of $28 \%$. 


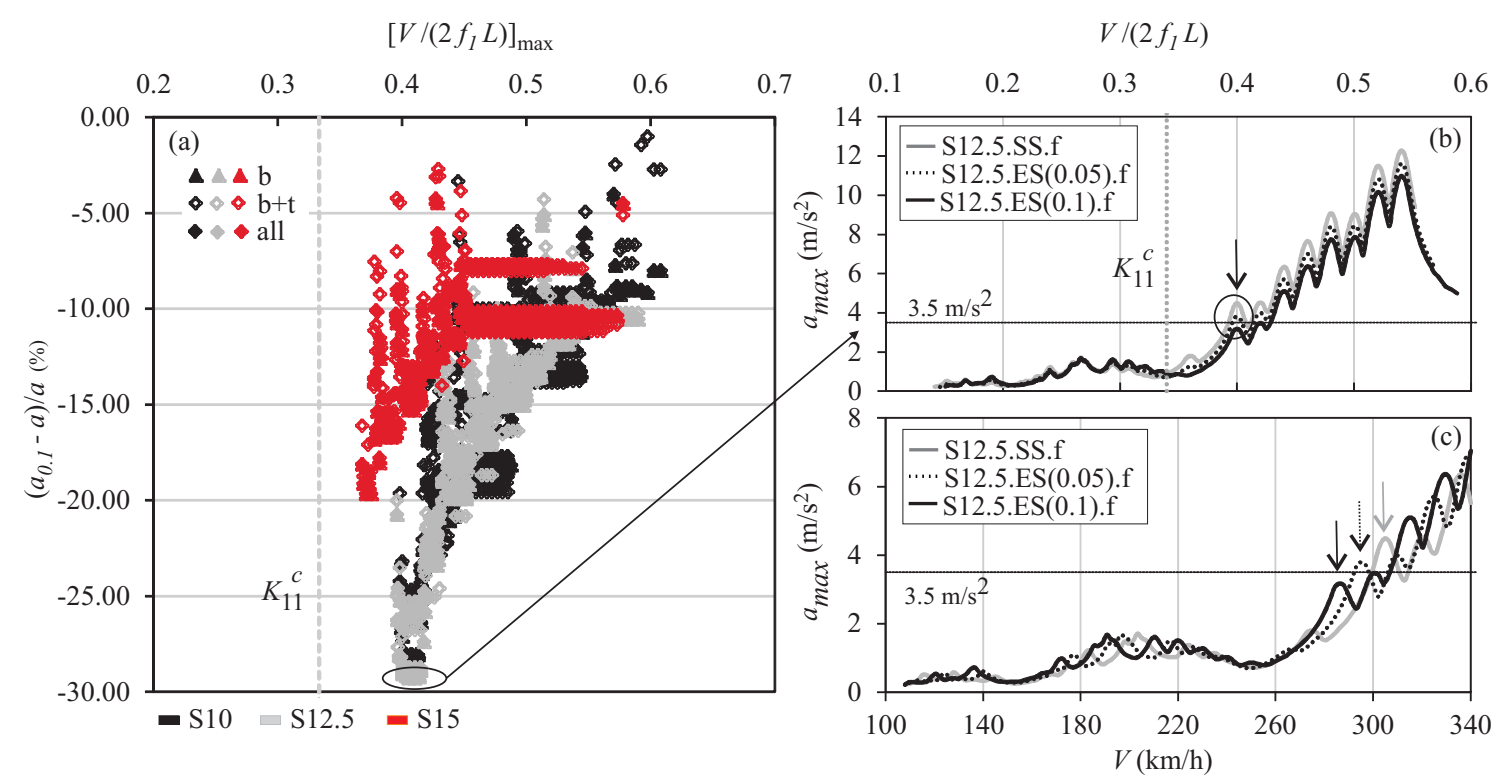

Figure 12: Influence of $\kappa$ in the prediction of the maximum acceleration levels for the slab bridges

$\left[V /\left(2 f_{l} L\right)\right]_{\max }$

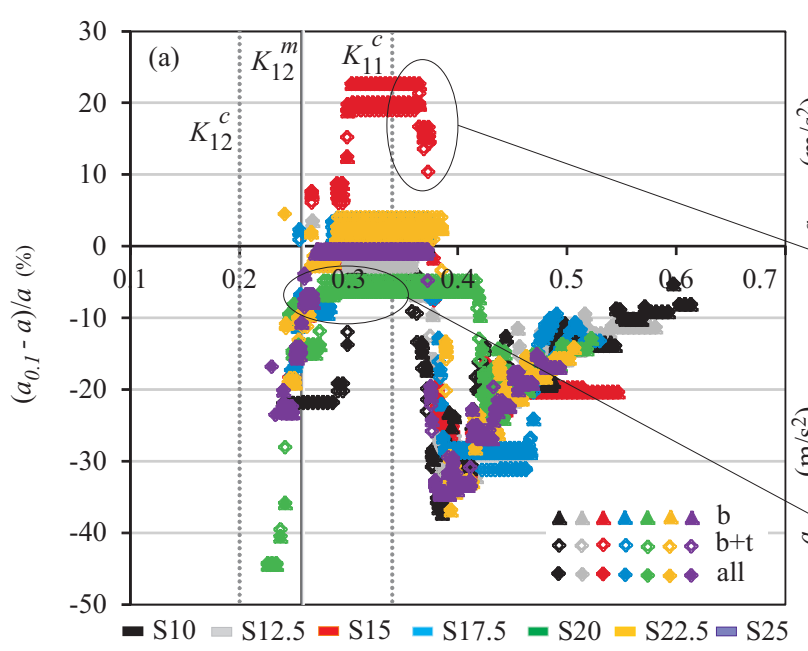

$V /\left(2 f_{l} L\right)$

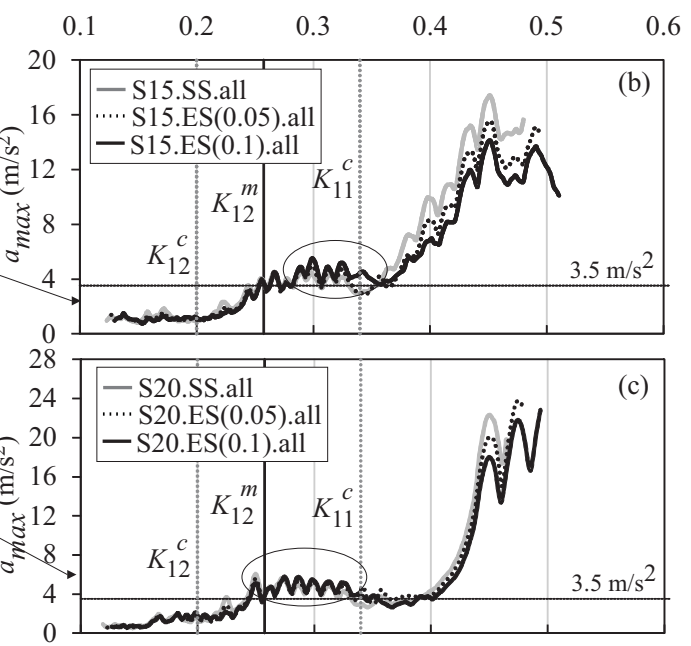

Figure 13: Influence of $\kappa$ in the prediction of the maximum acceleration levels for the slab bridges 


\subsubsection{Pre-stressed concrete girder bridges}

In the girder bridges the frequency differences of the first two mode shapes with respect to the rigid supported case are of the same order, and close to $6 \%$ when the most flexible elastic support is considered. Since the variations of natural frequencies lead to subsequent reductions of the resonance speeds, new acceleration peaks may appear in the range of speeds of interest when the elastomeric bearings are included in the numerical models. Nevertheless, as mentioned above, the dynamic analyses should be performed at speeds of circulation up to $20 \%$ higher than the maximum design velocity of the line, which is a considerably larger margin than the uncertainty in the determination of the resonance speeds due to the flexibility of the supports.

The influence of $\kappa$ in the prediction of the maximum acceleration levels has also been quantified as in the previous section, with the expression $\left(a_{0.1}-a\right) / a \times 100$. The results are shown in Fig. 13(a). As can be seen, in the girder bridges resonances leading to inadmissible levels of vertical acceleration can also be found at speeds right above the corresponding to the second cancellation $\left(K_{12}^{c}\right)$.

As the contribution of the first mode is very significant in the maximum dynamic response, the pattern of the resonance amplitudes observed in the E-S beam matches reasonably well the results shown in Fig. 13(a). Most of the differences found at speeds above $\left(K_{11}^{c}\right)$ are negative, meaning that the maximum acceleration peaks attain higher amplitudes when the supports are rigid. Some exceptions can be found in the plot, as in the case of the positive differences found in the girder bridge of $15 \mathrm{~m}$ of span; the acceleration envelopes including the contribution of all the modes prescribed by EC are shown in Fig. 13(b). These positive differences are only caused by the way in which the acceleration curves are compared. As can be seen in Fig 13 (b)in the range $V /\left(2 f_{1} L\right)=\left[\begin{array}{ll}0.3 & 0.38\end{array}\right]$ the resonance peak with the highest amplitude for $\kappa=0.1$ is the one occuring at $V /\left(2 f_{1} L\right)=0.3$, while the accelera- tion values for $\kappa=0$ are lower. At speeds above $K_{11}^{c}=1 / 3$, although it is clear from Fig 13. (b) that the acceleration envelope obtained with rigid supports (grey curve) predicts higher amplitudes than the one with $\kappa=0.1$ (black curve), the results in Fig[13(a) do not switch to negative values until the grey envelope overcomes the resonance peak at $V /\left(2 f_{1} L\right)=0.3$.

In the range of speeds between $K_{12}^{m}$ and $K_{11}^{c}$ inadmissible acceleration levels caused by resonances of the first bending mode can also be found, especially for the spans between 15 and $25 \mathrm{~m}$, where the introduction of the flexibility of the supports $(\kappa>0)$ in the numerical model raises the predicted resonance acceleration levels and, subsequently, most of the differences shown in Fig. 13(a) are positive. For reasons similar to the ones previously mentioned, some case studies do not follow the pattern predicted by Eq. (12): Fig. 13(c) shows a bridge of $20 \mathrm{~m}$ span in which a resonance peak at $V /\left(2 f_{1} L\right)=0.25$ predicted with $\kappa=0$, is causing that in the range $V /\left(2 f_{1} L\right)=[0.25$ 0.42] the corresponding markers in Fig. 13)(a) are negative instead. Finally, between $K_{12}^{c}$ and $K_{11}^{m}$ the markers in Fig. (a) are negative, which is also in accordance with Eq. (11).

In general Eqs. (11) and (12) will predict consistently whether the model with rigid supports is more conservative than the one with elastic supports or vice versa for each resonant peak. If the comparison includes every lower speed, the exceptions found here should be taken into account.

The results reveal that the influence of the supports flexibility on the resonance amplitudes is significant, differences up to $40 \%$ have been found when comparing the resonance amplitudes predicted under variations of the supports flexibility. However there is not a clear conclusion in what concerns the convenience of including the elastic supports in the numerical model for a safe prediction of the maximum acceleration levels, since the resonance speed plays an important role. Under high uncertainties in the estimation of the supports flexibility, considering 
both rigid and flexible supports for the prediction of the maximum acceleration levels is recommended.

\section{Conclusions}

With the increase of the speed of railway vehicles a number of conventional lines are adapted for the circulation at higher velocities and then, the dynamic simulation of existing bridges subjected to these new operating speeds is required to ensure traffic safety. In this contribution the dynamic behavior of several simply supported bridges of some of the typologies traditionally found covering short-to-medium span lengths in conventional lines has been studied, in a view to give a response to several critical aspects for the implementation of numerical models, which are the influence of both the transverse vibration modes and the flexibility of elastomeric bearings in the prediction of the maximum vertical acceleration levels in the deck. After the dynamic simulation of an extensive set of bridges under the passage of high-speed trains the following conclusions may be outlined:

- As it is known, girder bridges exhibit a poor behaviour at high speeds. This largely justifies why they are rarely found in most high-speed lines of more recent construction. This work shows that they may undergo inadmissible vertical accelerations at speeds even close to 200 $\mathrm{km} / \mathrm{h}$. The behaviour of slab bridges in terms of vertical acceleration at high speeds is better than the former typology. The speed of circulation in these bridges may attain $270 \mathrm{~km} / \mathrm{h}$ without experiencing excessive vibration problems. Although slab bridges experience a better performance in terms of vertical accelerations, other factors such as deck cracking due to the increase of the operating speed, may cause that fatigue problems be determinant for the dynamic performance and should be analysed.

- Planar beam models are useful for the verification of the Serviceability Limit State of vertical accelerations in slab bridges, since their dynamic behaviour is mainly governed by resonances of the first longitudinal bending mode and the contribution of other eigenforms is less significant. Regarding girder bridges, their dynamic behaviour in terms of vertical accelerations becomes inadmissible due to resonances of the first longitudinal bending mode as well, but also acceleration peaks caused by resonances of the first torsion mode and other threedimensional modes may attain significant levels. This is more noticeable as the span length and the supports flexibility increases. Beam-like numerical models may be a useful approach for a preliminary assessment of the maximum acceleration levels according to building codes such as EC, though not for an accurate prediction of the acceleration response in cases with very flexible supports.

- When the conditions established by EC1 to use planar models are met (i.e., frequency of the first torsion mode 1.2 times higher than the frequency of the first longitudinal bending mode, for non skewed beam or plate type decks on rigid supports), the contribution of the first torsion mode or other three-dimensional modes can be neglected even though their frequencies fall below the criteria recommended by EC (i.e., modes with frequencies up to the greater of (i) $30 \mathrm{~Hz}$, (ii) 1.5 times the frequency of the fundamental mode or (iii) the frequency of the third one, should be considered for an accurate mode superposition analysis according to EC).

- The introduction of the vertical flexibility of the elastomeric bearings in the numerical models does not guarantee a conservative prediction of the dynamic response, since it leads to variations of the resonance amplitudes that increase or diminish with the bearings vertical flexibility. Simple conditions have been provided to determine a priori the evolution of the resonances of the fundamental mode with the flexibility of the supports, and can be useful to ascertain wheter the uncertainty in the estimation of this parameter could be determinant for a 
safe prediction of the maximum acceleration levels.

\section{References}

[1] ERRI-D-214/RP9, Ponts Rails pour vitesses $>200 \mathrm{~km} / \mathrm{h}$. Rapport final, European Committee for Standardization, Brussels, 2000.

[2] L. Frỳba, Dynamic behaviour of bridges due to high-speed trains, Workshop Bridges for High-Speed Railways Porto (June 2004) 137-158.

[3] CEN, Eurocode: Basis of structural design. Annex A2: Application for bridges. Final version, European Committee for Standardization, Brussels, 2005.

[4] H. V. Vu, A. M. Ordóñez, B. H. Karnopp, Vibration of a double beam system, J Sound Vib 229 (4) (2000) 807 - 822.

[5] Z. Oniszczuk, Free transverse vibrations of elastically connected simply supported double-beam complex system, J Sound Vib 232 (2) (2000) $387-403$.

[6] M. D. Martínez-Rodrigo, J. Lavado, P. Museros, Dynamic performance of existing high-speed railway bridges under resonant conditions retrofitted with fluid viscous dampers, Eng Struct 32 (3) (2010) $808-828$.

[7] E. Moliner, P. Museros, M. D. Martínez-Rodrigo, Retrofit of existing railway bridges of short to medium spans for high-speed traffic using viscoelastic dampers, Eng Struct 40 (2012) 519 528.

[8] L. Frỳba, Vibration of solids and structures under moving loads, Thomas Telford, 1999.

[9] Y. B. Yang, C. L. Lin, J. D. Yau, D. W. Chang, Mechanism of resonance and cancellation for train-induced vibrations on bridges with elastic bearings, Journal of Sound and Vibration 269 (12) (2004) $345-360$.

[10] P. Museros, E. Alarcon, Influence of the second bending mode on the response of high-speed bridges at resonance, Journal of Structural Engineering 131 (3) (2005) 405 - 415.

[11] Y.-S. Lee, S.-H. Kim, Structural analysis of 3d high-speed trainbridge interactions for simple train load models, Vehicle System Dynamics 48 (2) (2010) 263-281.

[12] W. W. Guo, H. Xia, G. D. Roeck, K. Liu, Integral model for train-track-bridge interaction on the sesia viaduct: Dynamic simulation and critical assessment, Comput \& Structures 112,113 (2012) $205-216$.

[13] J. D. Yau, Y. S. Wu, Y. B. Yang, Impact response of bridges with elastic bearings to moving loads, J Sound and Vibration 248 (1) (2001) $9-30$.

[14] P. Museros, E. Moliner, M. D. Martínez-Rodrigo, Free vibrations of simply-supported beam bridges under moving loads:
Maximum resonance, cancellation and resonant vertical acceleration, J Sound Vib 332 (2) (2013) 326 - 345.

[15] D. J. Gorman, Free vibration analysis of beams and shafts, Wiley, 1975.

[16] I. A. Karnovsky, O. Lebed, Formulas for structural dynamics. Tables, graphs and solutions, McGrawHill, 2000.

[17] Y. B. Yang, J. D. Yau, L. C. Hsu, Vibration of simple beams due to trains moving at high speeds, Eng Struct 19 (11) (1997) $936-944$.

[18] E. Savin, Dynamic amplification factor and response spectrum for the evaluation of vibrations of beams under succesive moving loads, J Sound Vib 248 (2) (2001) 267 - 288.

[19] A. V. Pesterev, B. Yang, L. A. Bergman, C. A. Tan, Revisiting the moving force problem, J Sound Vib 261 (1) (2003) 75 - 91.

[20] C. A. Felippa, Refined finite element analysis of linear and nonlinear two dimensional structures, Ph.D. thesis, University of California, Berkeley (1966).

[21] M. D. Martínez-Rodrigo, J. Lavado, P. Museros, Dynamic performance of existing high-speed railway bridges under resonant conditions retrofitted with fluid viscous dampers, Eng Struct 32 (3) (2010) $808-828$.

[22] A. R. Cusens, R. P. Pama, Bridge deck analysis, John Wiley \& Sons, 1975.

[23] E. C. Humbly, Bridge deck behavior, JHalsted Press, a division of John Wiley \& Sons, 1976. 